\title{
Atypical Association of Autoimmune Limbic Encephalitis with Anti-NMDA Receptor Antibodies in a Young Male Patient: Clinical, Imaging, and Neuropsychological Characteristics
}

\author{
José Alberto Choreño-Parra ${ }^{a}$ Tania de la Rosa-Arredondo ${ }^{b}$ \\ André Garibay-Gracián c, d Ingrid Montes de Oca-Vargas ${ }^{c, e}$ \\ Deyanira Capi-Casillas $^{d, f} \quad$ Karla Valeria González-Pérez ${ }^{g}$ \\ Parménides Guadarrama-Ortiz ${ }^{a} \mathrm{~g}$
}

aDepartment of Neurosurgery, Centro Especializado en Neurocirugía y Neurociencias México (CENNM), Mexico City, Mexico; ${ }^{b}$ Department of Neuropsychology, Centro Especializado en Neurocirugía y Neurociencias México (CENNM), Mexico City, Mexico; 'Programa de Servicio Social en Investigación, Centro Especializado en Neurocirugía y Neurociencias México (CENNM), Mexico City, Mexico; 'dEscuela Nacional de Medicina y

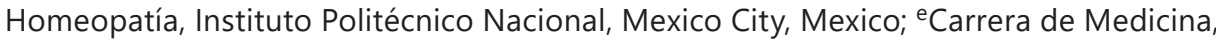
Facultad de Estudios Superiores (FES) Iztacala, Universidad Nacional Autónoma de México (UNAM), Talnepantla de Baz, Mexico; Internado Médico de Pregrado, Centro Especializado en Neurocirugía y Neurociencias México (CENNM), Mexico City, Mexico; 'Escuela de Medicina y Cirugía, Universidad Anáhuac Oaxaca, Oaxaca, Mexico

Keywords

Autoimmune encephalitis - Autoimmune limbic encephalitis - Anti-N-methyl-D-aspartate receptor antibodies $\cdot$ Neuropsychiatric symptoms $\cdot$ Noninfectious acute encephalitis

\begin{abstract}
The spectrum of autoimmune encephalitis (AE) encompasses several entities characterized by a variable frequency of psychiatric symptoms, cognitive dysfunction, focal deficits, and seizures. Although patients with AE can be categorized in specific syndromes, overlapping manifestations are also common. Furthermore, atypical correlations between clinical phenotypes and autoantibody profiles could occur in rare cases. Here, we report the rare case of a young adult man attending due to new-onset seizures and a history of memory loss, autonomic disturbances, headache, behavioral changes, and visual and olfactory hallucinations. The patient was subjected to a complete diagnostic approach that included a comprehensive laboratory workup, neuropsychological testing, electroencephalogram, cerebrospinal fluid (CSF) analy-
\end{abstract}


sis, brain MRI, and positron emission tomography/computed tomography scan that revealed a functional and structural compromise of the bilateral medial temporal lobes. Together with the clinical manifestations of the patient, these findings were compatible with the diagnosis of autoimmune limbic encephalitis (ALE). Strikingly, further analysis of the CSF showed autoantibodies against the $\mathrm{N}$-methyl-D-aspartate (NMDA) receptor. We found very few cases of the co-occurrence of anti-NMDA receptor antibodies and nonparaneoplastic ALE in the literature, especially in male patients. Our report exemplifies the complicated differential diagnosis of ALE and adds clinical information of the association with anti-NMDA receptor antibodies.

(C) 2021 The Author(s).

Published by S. Karger AG, Basel

\section{Introduction}

Autoimmune encephalitis (AE) is currently recognized as a common cause of brain inflammation in patients with subacute noninfectious encephalopathy. This disorder encompasses a range of clinical syndromes characterized by a variable frequency of psychiatric symptoms, working memory impairment, altered mental status, focal deficits, and seizures. Such manifestations are frequently associated with autoantibodies against intracellular antigens, synaptic receptors, ion channels, and other cell-surface proteins. Among these, IgG antibodies against the glycine subunit (GluN1) of the N-methyl-Daspartate (NMDA) receptor occur in a substantial number of patients with AE, primarily as a paraneoplastic syndrome affecting young women with an underlying ovarian teratoma [1].

Autoimmune limbic encephalitis (ALE) is one of the best-defined clinical syndromes of the spectrum of AE that results from the involvement of the medial temporal lobes [2]. The cases of this disorder, unlike those with global AE, are commonly associated with antibodies against the onconeuronal antigens $\mathrm{Hu}$ and $\mathrm{Ma} 2[3,4]$ and cell-surface antibodies like leucine-rich glioma-inactivated 1 [5], type b gamma-aminobutyric acid receptor [6], and $\alpha$-amino-3-hydroxy-5-methyl-4-isoxazolepropionic acid receptor [7]. Very little evidence exists on the co-occurrence of anti-NMDA receptor antibodies and brain inflammation restricted to the limbic system in patients with AE [1, 8-11]. Indeed, although anti-NMDA receptor encephalitis and ALE have been proposed as different recognizable syndromes [2], the literature is not clear about the possible overlap between these disorders. Interestingly, rare associations of anti-NMDA receptor antibodies with other forms of AE like acute disseminated encephalomyelitis have been reported [12]. Notably, there are very few cases of an anti-NMDA receptor ALE, and most of them occur as a paraneoplastic phenomenon in females $[9,11,13]$. Since only a few reports of nonparaneoplastic anti-NMDA receptor ALE have been observed in men $[9,11]$, the clinical phenotype of this association is not well characterized.

Here, we describe the rare case of a young male patient who presented with a history of psychiatric symptoms, short-term memory deficits, and seizures. Our diagnostic approach revealed psychiatric manifestations of the limbic system, epileptic activity, imaging abnormalities of the brain highly restricted to the bilateral medial temporal lobes, anti-NMDA receptor antibodies in the cerebrospinal fluid (CSF), and no evidence of an underlying tumor. Our report exemplifies the complicated differential diagnosis of $\mathrm{AE}$ and adds clinical information about the phenotype of nonparaneoplastic anti-NMDA receptor ALE in men.

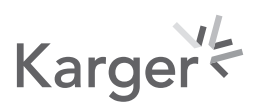




\section{Case Report/Case Presentation}

A 44-year-old right-handed man with a 19-year history of generalized anxiety disorder attended our center after a generalized tonic-clonic seizure, postictal status, and secondary head trauma. Two years before, the patient developed a tension-type headache accompanied by short-term memory loss, behavioral changes, irritability, deja vu, and visual and olfactory hallucinations. He was diagnosed with temporal lobe epilepsy, for which he received unspecified management at another hospital achieving minimal improvement. The patient had a high education level and worked as the manager of an important car rental global company. During the last year before admission, he presented a marked cognitive decline characterized by intermittent and progressive episodes of affective flattening, apathy, and exacerbated memory loss. Each crisis was always accompanied by autonomic instability symptoms, such as dizziness, paleness, tremor, postural instability, and drowsiness. These manifestations severely affected his professional activities due to constant omissions and oversights. Also, he developed a lack of adherence to his managerial obligations and an inability to give talks, even when one of his most remarkable skills was public speaking.

His past family history was not relevant, and he denied any toxic exposure, drug abuse, or contact with wild animals. On admission, the patient was alert and oriented to person, place, and time. His vital signs were within normal ranges. The physical examination did not reveal any abnormality in cranial/peripheral nerves, muscle strength, deep tendon reflexes, and plantar reflexes. His gait and the finger-to-nose test were normal, and meningeal signs were absent. The results of the initial laboratory workup showed a hemoglobin of $16.3 \mathrm{~g} / \mathrm{dL}$, erythrocytes $5.3\left(10^{9} / \mathrm{L}\right)$, platelets $252\left(10^{9} / \mathrm{L}\right)$, glucose $110 \mathrm{mg} / \mathrm{dL}$, white blood cells 14.1 $\left(10^{9} / \mathrm{L}\right)$, neutrophils $12.2\left(10^{9} / \mathrm{L}\right)$, lymphocytes $1.5\left(10^{9} / \mathrm{L}\right)$, and monocytes $0.2\left(10^{9} / \mathrm{L}\right)$. Also, thyroid, liver, and renal function parameters as well as the urinalysis, metabolic panel, lipid panel, prothrombin time, partial thromboplastin time, international normalized ratio, fibrinogen, procalcitonin, lactate dehydrogenase, MB fraction of creatine phosphokinase, erythrocyte sedimentation rate, C-reactive protein, rheumatoid factor, and serum levels of calcium, magnesium, sodium, potassium, and chloride were normal.

Due to the history of cognitive abnormalities and the presence of new-onset seizures associated with leukocytosis and neutrophilia, we suspected a parainfectious or immunemediated disorder. Therefore, the patient underwent additional analyses. An electroencephalogram (EEG) recording at rest revealed sharp-wave complexes in the bilateral frontal and temporal lobes. Meanwhile, the CSF analysis showed normal opening pressure, glucose 54 $\mathrm{mg} / \mathrm{dL}$, proteins $54.2 \mathrm{mg} / \mathrm{dL}$, normal adenosine deaminase, and no cells, oligoclonal bands, or bacterial growth. MRI of the brain showed no brain edema, hemorrhage, infarction, and tumor. Strikingly, an increased T2 signal was observed in the bilateral medial temporal lobes (Fig. 1), which correlated with a decreased uptake of 18 fluorodeoxyglucose $\left({ }^{18} \mathrm{FDG}\right)$ in the same areas in the positron emission tomography/computed tomography (PET/CT) scan of the brain (Fig. 2).

The complexity of the case prompted us to consult neuropsychologists of our center, which further assessed for specific deficits in several cognitive domains using a comprehensive battery of neuropsychological tests. These included the Montreal Cognitive Assessment (MoCA) test, Hamilton Anxiety Rating Scale (HAM-A), Beck Depression Inventory (BDI), Complutense Verbal Learning Test(TAVEC), Wechsler Memory Scale (WMS), NEUROPSI (Np) test, token test, Wisconsin Card Sorting Test (WCST), Tower of London Drexel University version (TOL DX) test, Barcelona test (Bt), and Paced Auditory Serial Addition Test (PASAT). The patient obtained a MoCA score of 25, indicating mild cognitive impairment. Also, he scored 38 in the HAM-A and 26 in the BDI, compatible with moderate anxiety and moderateto-severe depression, respectively. This evaluation also showed dysfunction in selective

\section{Karger'}



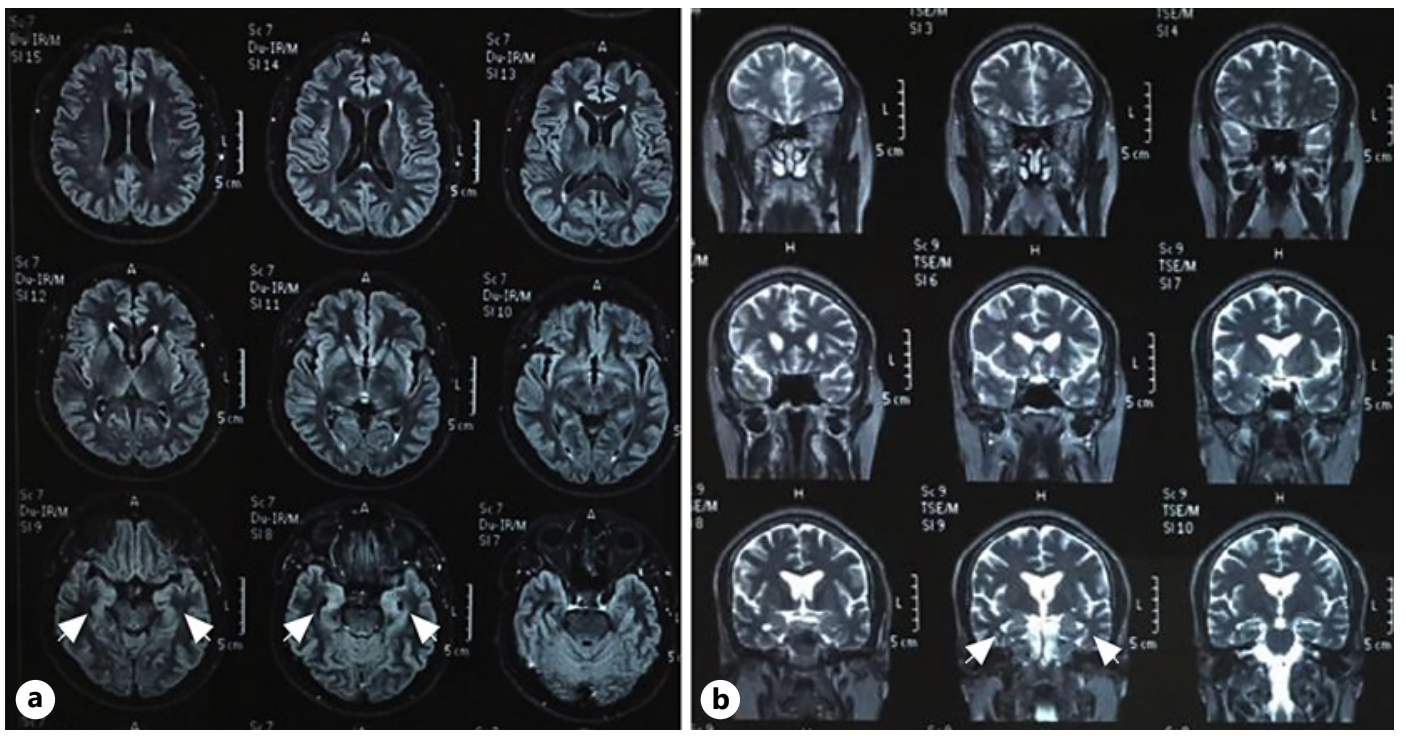

Fig. 1. Axial T2-weighted FLAIR (a) and coronal T2-weighted brain MRI (b) images showing atrophy and hyperintense signal in the bilateral medial temporal lobes (white arrows). FLAIR, fluid-attenuated inversion recovery; MRI, magnetic resonance imaging.

attention and alterations in verbal working memory due to difficulties in encoding and retrieval from the memoranda storing that benefit from the use of facilitators like cues and intrusions.

The spectrum of cognitive disorders presented by the patient, together with the laboratory and imaging findings, led us to the suspicion of probable ALE, which was confirmed by analyzing CSF levels of autoantibodies. Surprisingly, the results of this analysis showed elevated titers of anti-NMDA receptor antibodies. Since these antibodies are often related to underlying tumors, we performed a thoracoabdominal CT scan, which was negative for tumor and lymphadenopathy. The patient received intravenous methylprednisolone and phenytoin, as well as oral olanzapine, fluoxetine, and clonazepam, showing significant clinical improvement. He was discharged after 10 days of hospitalization and scheduled for additional evaluations at our outpatient clinic. However, the patient never attended his follow-up appointments. After several failed attempts to contact the patient, his relatives informed us that he presented a new exacerbation of his clinical manifestations, developing a severe cognitive impairment that made him leave his home and live on the streets. Since then, his family has been searching for him in homeless shelters. The legal guardian of the patients provided written informed consent for the publication of this case report.

\section{Discussion/Conclusion}

$\mathrm{AE}$ is a diagnostic challenge for clinicians due to its unspecific manifestations that are often hard to differentiate from infectious encephalitis or true neuropsychiatric disorders. Indeed, an extensive list of diseases can mimic AE, including infections, delirium, psychosis, illicit drug abuse, drug toxicity, stroke, epilepsy, Creutzfeldt-Jakob disease, brain tumors, early-onset Alzheimer disease, mitochondrial diseases, and inborn metabolism disorders. Furthermore, a group of clinically recognizable syndromes has been described among patients with $\mathrm{AE}$, adding complexity to the differential diagnosis of the disease. These syndromes 

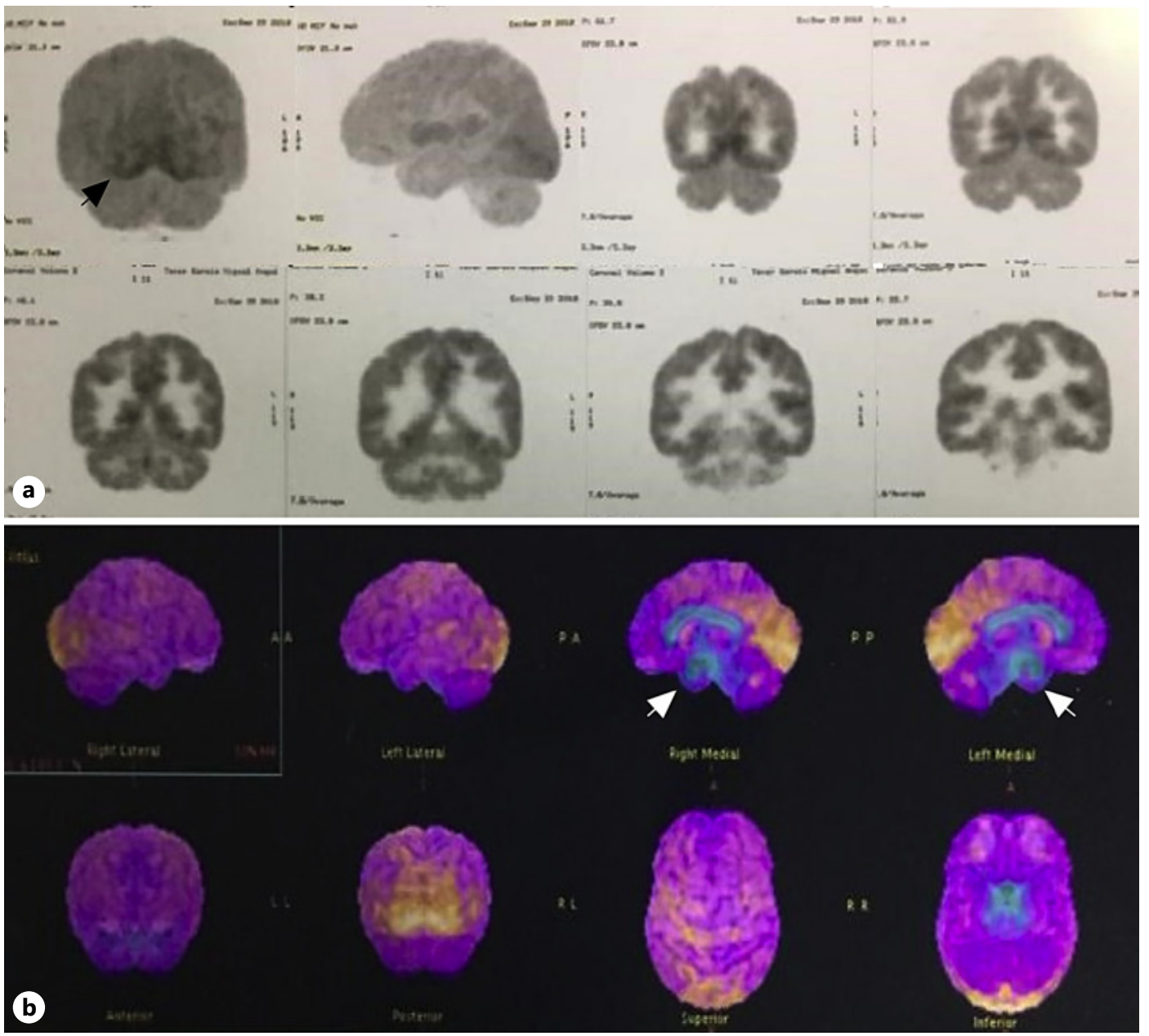

Fig. 2. Brain ${ }^{18}$ FDG-PET/CT scan (a) and 3D-SSP uptake maps (b) showing decreased uptake of ${ }^{18}$ FDG at the medial temporal lobes (arrows). ${ }^{18}$ FDG-PET/CT, 18 fluorodeoxyglucose positron emission tomography/ computed tomography.

include the anti-NMDA receptor encephalitis, acute disseminated encephalomyelitis, ALE, and Bickerstaff's brainstem encephalitis [2]. Although some characteristics might distinguish between specific $\mathrm{AE}$ forms, most patients do not present a well-defined syndrome.

In the past, most diagnostic criteria did not consider a possible autoimmune origin of acute encephalitis [14]. Thus, the diagnosis of AE was commonly reserved for those patients with negative testing for viral infections and strongly depended on blood/CSF antibody testing and response to immunosuppressive therapy. As antibodies are not always available, several AE criteria have been recently proposed based on clinical manifestations, cognitive deficits, psychiatric symptoms, CSF analysis, EEG, brain MRI, and ${ }^{18}$ FDG-PET/CT scan [2]. These criteria consider three levels of evidence for AE: possible, probable, and definitive. After exclusion of other causes, a case of possible AE is defined as a subacute onset of shortterm memory deficits, altered mental status or psychiatric manifestations, and at least one of the following characteristics: new focal neurological findings, unexplained seizures, CSF pleocytosis, and MRI abnormalities suggestive of encephalitis.

The category of probable includes diagnostic criteria more specific for each recognizable syndrome. For instance, the presence of abnormalities restricted to the medial temporal lobes in imaging studies of the brain is essential to define a case of probable ALE. In contrast, 
diffuse lesions of the cerebral white matter or deep gray matter abnormalities are diagnostic criteria for probable acute disseminated encephalomyelitis, whereas an underlying ovarian or systemic teratoma in young women could support the diagnosis of probable anti-NMDA receptor AE. The presence of autoantibodies in the blood or CSF is crucial as they confirm a definitive diagnosis and provide information about the frequency of tumor association, prognosis, and treatment response [2].

Despite these advances, several patients are not identified early, and thus they do not receive an opportune treatment. This problem is well illustrated by the case described here, which also exemplifies the problematic and sometimes delayed differential diagnosis of $\mathrm{AE}$. Indeed, our case presented several atypical characteristics that broadened the list of diagnostic possibilities. First, our patient was a young adult man otherwise physically healthy. This fact is striking as, in general, AE tends to affect primordially women, in some instances with a three-fold higher frequency than men, as for the case of anti-NMDA receptor AE [15, 16]. Second, the patient attended our center after two years of symptoms onset. This chronic disease course opened the possibility for a true psychiatric disorder, brain tumor, early-onset dementia, and even temporal lobe epilepsy, as he was wrongly categorized in another hospital. All these disorders were ruled out by our complete and sophisticated diagnostic approach, which allowed us to confirm the disease with high certainty and provide the patient with a definitive explanation for his long-lasting and debilitating symptoms that would otherwise remain obscure.

Hence, a strength of this report is that our clinical evaluation of the case included an inquiry into the patient's history, physical examination, neuropsychological assessment, routine blood tests, CSF analysis, EEG, brain MRI, ${ }^{18}$ FDG-PET/CT scan, and CSF testing for autoantibodies. Some of these tools were of great utility for the case, and therefore they deserve further mentioning. For example, the EEG and imaging studies helped us to identify the area of the brain affected by the disease. Similarly, the battery of neuropsychological tests applied to our patient and the anatomic-physiological integration of their results allowed us to delimitate manifestations to the limbic system. In this regard, although there are several descriptions of the psychiatric disturbances of AE, only a few reports have objectively evaluated such abnormalities using validated neuropsychological tests [17]. Of note, we tested for autoantibodies in the CSF, as it is known that this approach yields more accurate results than the analysis of peripheral blood [18, 19].

Interestingly, we found high titers of anti-NMDA receptor antibodies in the CSF of our patient. To our knowledge, there are very few reports of the occurrence of these antibodies in patients with ALE, especially in males without underlying malignancies [9, 11, 13]. For instance, Henry et al. [13] reported a case of a young female patient with an ovarian teratoma that developed an anti-NMDA receptor ALE syndrome characterized by memory decline, psychiatric manifestations, and seizures. Meanwhile, Sarkis et al. [9] found that in a series of 16 cases of nonparaneoplastic ALE, only 5 patients had detectable levels of anti-NMDA receptor antibodies, including 2 young adult males. Interestingly, a common manifestation in all patients with nonparaneoplastic anti-NMDA receptor ALE was the presence of behavioral changes, which coincides with the clinical manifestations observed in our case [9]. Similarly, in another study, Shojima et al. [11] reported 12 cases of anti-NMDA receptor ALE, from which 11 patients were young women with underlying ovarian teratomas and only 1 man that presented with catatonia. Furthermore, only one out of 18 cases reported by Baumgartner et al. [8] showed a correlation between anti-NMDA receptor antibodies and mesiotemporal abnormalities in the brain MRI, but the investigators did not provide additional clinical information of the case.

In contrast, several other cases have been erroneously identified as anti-NMDA receptor ALE. Khadem et al. [1] reported a case of anti-NMDA receptor limbic encephalitis. However,

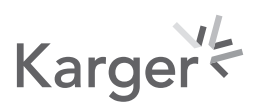


the clinical characteristics of the patient somewhat resembled a global anti-NMDA AE syndrome, and the imaging studies of the brain did not show abnormalities of the medial temporal lobes. Likewise, Muñoz et al. [10] described a case of anti-NMDA receptor ALE. However, the manifestations of the patient were not consistent with limbic encephalitis, and the authors could not demonstrate the presence of autoantibodies. These studies, together with our report, indicate that the occurrence of anti-NMDA receptor ALE is a very infrequent entity observed among males without underlying malignancies. Also, the current evidence remarks that there is a high grade of confusion about the diagnosis and classification of $\mathrm{AE}$ among physicians. In fact, we also faced a problematic dilemma as our case presented clinical and radiological features consistent with ALE but also overlapped with the definition of antiNMDA receptor AE. This confusion suggests that the current diagnostic criteria for AE could improve. Despite this, and based on the prevailing definitions, we consider ours as one of the few reported cases of definitive ALE associated with anti-NMDA receptor antibodies.

Finally, the chronicity of our case makes this report a useful contribution to the field of $\mathrm{AE}$, as it demonstrates the long-lasting nature of the disorder and illustrates some specificities of late disease stages. For instance, our observations suggest that CSF testing can detect autoantibodies even a long time after symptoms onset. Our case might also indicate that patients with chronic manifestations could not respond well to intravenous steroids, although proper longitudinal studies must validate this conclusion.

\section{Acknowledgments}

The authors acknowledge Daniel Prieto-Rivera and Patricia E. Rodríguez-Muñoz, for their critical reading and comments on the manuscript.

\section{Statement of Ethics}

The patient provided written informed consent for the publication of this case report and any accompanying images in accordance with the World Medical Association Declaration of Helsinki. The study is exempt from ethics committee approval because of its single-case design.

\section{Conflict of Interest Statement}

The authors have no conflicts of interest to declare.

\section{Funding Sources}

The current study did not receive financial support.

\section{Author Contributions}

T.R.-A., A.G.-G., I.M.O.-V., and P.G.-O provided medical care to the patient. J.A.C.-P., A.G.-G., I.M.O.-V., D.C.-C., and K.V.G.-P retrieved clinical data of the case. J.A.C.-P. and P.G.-O drafted the manuscript. All the authors read and approved the final version of the manuscript. 


\section{Data Availability Statement}

All clinical data analyzed during this report are included in this article. Further enquiries can be directed to the corresponding author.

\section{References}

1 Khadem GM, Heble S, Kumar R, White C. Anti-N-methyl-D-aspartate receptor antibody limbic encephalitis. Intern Med J. 2009 Jan;39(1):54-6.

2 Graus F, Titulaer MJ, Balu R, Benseler S, Bien CG, Cellucci T, et al. A clinical approach to diagnosis of autoimmune encephalitis. Lancet Neurol. 2016 Apr;15(4):391-404.

3 Alamowitch S, Graus F, Uchuya M, Reñé R, Bescansa E, Delattre JY. Limbic encephalitis and small cell lung cancer. Clinical and immunological features. Brain. 1997 Jun;120(Pt 6):923-8.

4 Dalmau J, Graus F, Villarejo A, Posner JB, Blumenthal D, Thiessen B, et al. Clinical analysis of anti-Ma2-associated encephalitis. Brain. 2004 Aug; 127(Pt 8):1831-44.

5 Lai M, Huijbers MG, Lancaster E, Graus F, Bataller L, Balice-Gordon R, et al. Investigation of LGI1 as the antigen in limbic encephalitis previously attributed to potassium channels: a case series. Lancet Neurol. 2010 Aug; 9(8):776-85.

6 Lancaster E, Lai M, Peng X, Hughes E, Constantinescu R, Raizer J, et al. Antibodies to the GABA(B) receptor in limbic encephalitis with seizures: case series and characterisation of the antigen. Lancet Neurol. 2010 Jan; $9(1): 67-76$.

7 Lai M, Hughes EG, Peng X, Zhou L, Gleichman AJ, Shu H, et al. AMPA receptor antibodies in limbic encephalitis alter synaptic receptor location. Ann Neurol. 2009 Apr;65(4):424-34.

8 Baumgartner A, Rauer S, Mader I, Meyer PT. Cerebral FDG-PET and MRI findings in autoimmune limbic encephalitis: correlation with autoantibody types. J Neurol. 2013 Nov;260(11):2744-53.

9 Sarkis RA, Nehme R, Chemali ZN. Neuropsychiatric and seizure outcomes in nonparaneoplastic autoimmune limbic encephalitis. Epilepsy Behav. 2014 Oct;39:21-5.

10 Muñoz D, Hernández DL, Giraldo N. Limbic encephalitis with phenotypic NMDA receptor antibodies in patients with de novo diagnosis of Systemic Lupus Erythematosus. Case report. Colombian J Anesthesiology. 2017;45: 59-65.

11 Shojima Y, Nishioka K, Watanabe M, Jo T, Tanaka K, Takashima H, et al. Clinical characterization of definite autoimmune limbic encephalitis: a 30-case series.Intern Med. 2019;58(23):3369-78.

12 Liu J, Yi H, Xu L, Li M, Wang X, Peng F-H. A case of anti-NMDA receptor encephalitis with ADEM-like clinical/ MR findings. Neuroimmunol Neuroinflamm. 2016;3:257-9.

13 Henry C, Husson $\mathrm{H}$, de Broucker T. [Autoimmune limbic encephalitis with anti-NMDA receptor antibodies and ovarian teratoma: a treatable form of paraneoplastic limbic encephalitis]. Rev Neurol. 2009 Jan;165(1):70-5.

14 Ball R, Halsey N, Braun MM, Moulton LH, Gale AD, Rammohan K, et al. Development of case definitions for acute encephalopathy, encephalitis, and multiple sclerosis reports to the vaccine: adverse event reporting system. J Clin Epidemiol. 2002 Aug;55(8):819-24.

15 Wingfield T, McHugh C, Vas A, Richardson A, Wilkins E, Bonington A, et al. Autoimmune encephalitis: a case series and comprehensive review of the literature.QJM. 2011;104(11):921-31.

16 Viaccoz A, Desestret V, Ducray F, Picard G, Cavillon G, Rogemond V, et al. Clinical specificities of adult male patients with NMDA receptor antibodies encephalitis. Neurology. 2014 Feb 18;82(7):556-63.

17 Finke C, Kopp UA, Prüss H, Dalmau J, Wandinger KP, Ploner CJ. Cognitive deficits following anti-NMDA receptor encephalitis. J Neurol Neurosurg Psychiatry. 2012 Feb;83(2):195-8.

18 Titulaer MJ, McCracken L, Gabilondo I, Armangué T, Glaser C, Iizuka T, et al. Treatment and prognostic factors for long-term outcome in patients with anti-NMDA receptor encephalitis: an observational cohort study. Lancet Neurol. 2013 Feb;12(2):157-65.

19 Gresa-Arribas N, Titulaer MJ, Torrents A, Aguilar E, McCracken L, Leypoldt F, et al. Antibody titres at diagnosis and during follow-up of anti-NMDA receptor encephalitis: a retrospective study. Lancet Neurol. 2014 Feb; 13(2):167-77. 